Short report

\title{
Mini-invasive technique of implanting the first domestic wireless epicardial pacemaker with a MEMS-converter
}

\author{
Olga L. Bockeria, Mikhail B. Biniashvili, Tatyana G. Le, Anna S. Satyukova, Daniyar K. Zhiengaliev, \\ Vladimir A. Shvartz
}

Bakulev National Medical Research Center for Cardiovascular Surgery, Moscow, Russia

Received 25 March 2020, Revised 21 April 2020, Accepted 28 April 2020

(C) 2020, Bockeria O.L., Biniashvili M.B., Le T.G., Satyukova A.S., Zhiengaliev D.K., Shvartz V.A

(C) 2020, Russian Open Medical Journal

\begin{abstract}
This article describes a minimally invasive surgical technique for implanting the pacemaker with a microelectromechanical system (MEMS) converter of kinematic energy into electrical energy in patients with life-threatening rhythm disorders. This kind of technique is recommended for patients with cardiac pathology who are preparing for surgical treatment of the main pathology with simultaneous implantation of the pacemaker with MEMS-converter of kinematic energy into electrical energy. Implantation of the pacemaker should be performed in the most energy-efficient zone of the epicardium, determined in advance by the method of tissue echocardiography.
\end{abstract}

Keywords: mini-invasive technique, wireless pacemaker, epicardial pacemaker, MEMS-converter.

Cite as Bockeria OL, Biniashvili MB, Le TG, Satyukova AS, Zhiengaliev DK, Shvartz VA. Mini-invasive technique of implanting the first domestic wireless epicardial pacemaker with a MEMS-converter. Russian Open Medical Journal 2020; 9: e0206.

Correspondence to Tatyana G. Le. Address: 135, Rublevskoe shosse, Moscow, 121552, Russia. E-mail: tanya co@mail.ru.

\section{Introduction}

The implantation of the pacemaker is usually the only salvation for patients with severe rhythm disorders such as life-threatening bradyarrhythmias. The heart rate disorders, as well as any abnormalities of the heart rhythm seriously affect the state of the entire organism and in some particularly severe cases can lead to death. Therefore, often, if it is impossible to normalize the heart rate with medications, an artificial pacemaker should be implanted [1].

The pacemaker is the device which has a power source and one or more electrodes. This system works until the battery is depleted. Battery life depends on various factors - the manufacturer, material of the battery, the mode of functioning of the device, but on average they serve for about 5-10 years. After the battery is depleted, the device must be replaced [2].

Over time, the appearance, weight-size properties and method of implantation of the pacemaker changed. They became smaller and more compact, and it became possible to introduce the electrodes to the heart through the catheter using a vascular access, the so-called endocardial pacemaker. However, the problem of depletion of the power source still exists as well as inevitability of repeated interventions. In addition, the wire part the electrode-implanted in the endocardium of the heart can wear out, bend, and break with atypical sudden movements and injuries, and in the case of such complications, it is also necessary to replace the wire part of the pacemaker. In addition to mechanical complications related to the integrity of the electrodes, there are also complications such as thrombosis of the electrode part of the pacemaker and infection. After that, the obvious question arises, is it possible to design a wireless pacemaker to avoid all of the above complications? [2].

Thus, a group of authors invented a domestic single-chamber and dual-chamber epicardial pacemaker with microelectromechanical system (MEMS) converter of kinematic energy of the heart into electrical energy, which consists of the following components:

i) epicardial pacemaker with MEMS-converter of kinematic energy of the heart into electrical energy;

ii) electrode part for providing various modes of pacing, it has a screw-in part, which is also a fixing part;

iii) electrode for atrial stimulation, which is a conductive part (for a dual-chamber pacemaker)

Single- and dual-chamber epicardial pacemaker with MEMSconverter of kinematic energy of the heart into electrical energy designed to provide the electrotherapy for patients with complex rhythm and conduction disorders, including those after cardiac surgery.

As for any medical device there are indications for implantation of the epicardial single- and dual-chamber pacemaker with MEMS-converter.

Indications for implantation of a single- and dual-chamber epicardial pacemaker with MEMS-converter

The indications for implantation of an epicardial pacemaker are all kinds of bradiarrhythmias.

1) Sinus node dysfunction: 
a) sinus node weakness syndrome,

b) sinoatrial block,

c) sinus bradycardia less than 40 beats per minute;

2) Atrioventricular block.

It should be noted that the implantation of a single-chamber pacemaker with MEMS-converter is preferable for patients who have atrial tachyarrhythmias (atrial fibrillation, atrial flutter, paroxysmal atrial tachycardia) against the background of bradycardia.

Usually these are age-related patients with degenerative changes in the sinus node, or patients with coronary heart disease who have developed sinus node dysfunction due to ischemia. These patients sooner or later end up in a cardiosurgical hospital.

In contrast to patients with atrial tachyarrhythmias, for whom dual-chamber pacemaker with MEMS-converter is undesirable, because the atrial electrode has a sense-function, and making detection of a signal from the atria during arrhythmia, will impose the same number of beats on the ventricles, which can lead to ventricular fibrillation, patients without tachyarrhythmias are indicated to a dual-chamber pacemaker with a MEMS-converter [3].

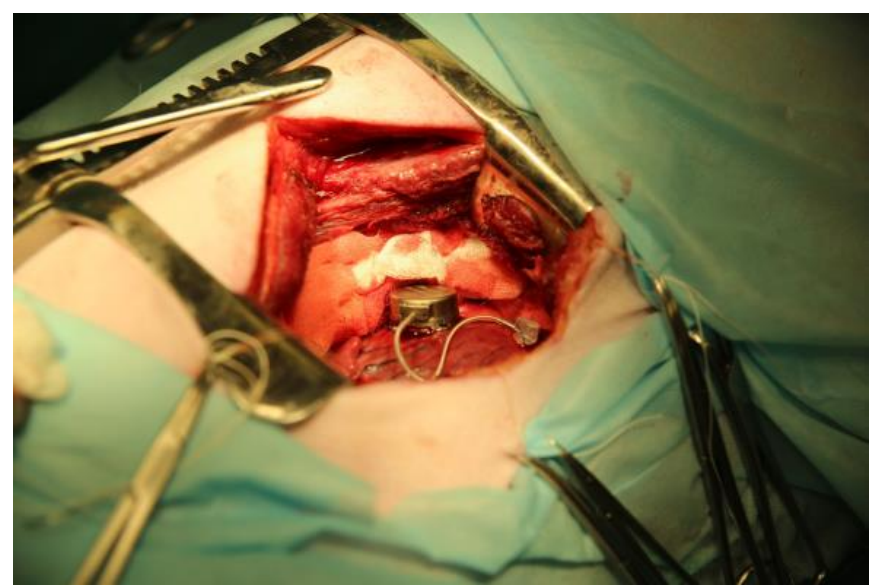

Figure 1. Dual-chamber pacemaker with a MEMS-converter on the LV epicardium and the electrode on the wall of the left atrium ear.

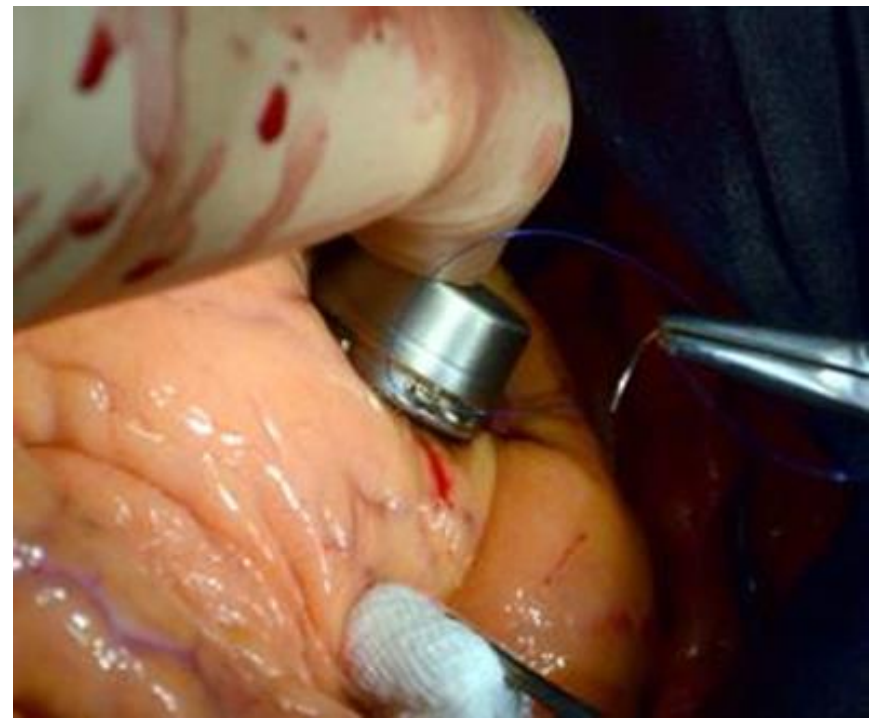

Figure 2. Fixation of the pacemaker with a MEMS-converter on the surface of the left ventricle using the "sewing" technique.

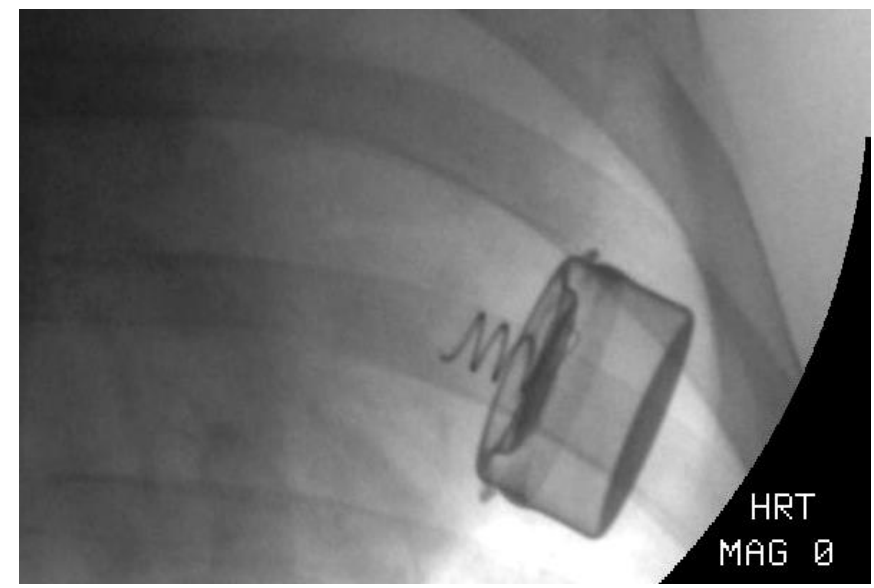

Figure 3. View of the fixed single-chamber pacemaker with a MEMS converter on the surface of the left ventricle on fluoroscopy.

The dual-chamber pacemaker with MEMS-converter has a physiological stimulation. The impulse spreads from the atria to the ventricles, which is much better tolerated clinically by patients and over time causes less signs of heart failure [4].

To provide epicardial stimulation with single- and dual-chamber pacemaker, it is necessary to provide proper access to the surface of the heart, and is possible only during open-heart surgery.

\section{Surgical method of implantation}

The access is performed by the left lateral mini-thoracotomy. The patient is in the right semi-lateral position. A minithoracotomy is performed in the fourth or fifth intercostal space on the left side through a skin incision along the mid-axillary line from 3 to $5 \mathrm{~cm}$ long. The left lung is pushed aside and the pericardium is carefully opened without damaging the diaphragmatic nerve. Then the pericardium is fixed and the heart carefully turns to the right, which allows you to adequately visualize the side and back walls of the left ventricle (LV). The epicardial electrode with a steroid coating is fixed in an adequate position in the most energy-efficient zone, previously determined by the method of tissue echocardiography, and then the parameters are measured from the electrode.

For implantation of a single-chamber pacemaker, the technique of "screwing" is used, which includes the following actions: initially, with the help of tampon holders, the intervascular section of the LV wall is stabilized, then the pacemaker with MEMS-converter is brought to the LV wall with an arrangement of $90^{\circ}$ to the LV wall. The pacemaker with the MEMSconverter is fixed and positioned in the inter-vascular zone. Then the pacemaker with the MEMS-converter is carefully screwed into the epicardium and then into the LV myocardium by 2.5 turns counterclockwise.

For implantation of a dual-chamber pacemaker with a MEMSconverter on the LV epicardium, two different methods are simultaneously used: "screwing" the fixing electrode into the epicardium of the left ventricle, then "screwing" the second electrode into the wall of the left atrium ear (Figure 1), and finally "sewing" the pacemaker itself to the LV epicardium for fixing loops (Figure 2).

The technique of "sewing" includes the following actions: using a needle holder and tweezers, there are two stitches are applied to 
the LV epicardium in the area of the connectors for fixing the pacemaker with the MEMS-converter, and then the nodes are tied, capturing the connectors for fixing the device. When carefully pulling the proximal part of the pacemaker with the MEMSconverter, the strength of the fixation is determined. Then the pericardium is reduced by separate 2-3 nodal sutures (atraumatic Dacron thread $20 \mathrm{~mm}$ ), then comes suturing of the pericardium, installation of pleural drainage and suturing of the chest. Figure 3 shows the view of the fixed single-chamber pacemaker with a MEMS-converter on the surface of the left ventricle on fluoroscopy.

Comparison of electrode implantation methods: epicardial and endocardial

Despite the invasive nature of surgery and longer hospital stays, including the intensive care unit, the surgical technique avoids the potentially dangerous risks of the transvenous approaches.

N. Mair et al. in 2005 compared the results of treatment of patients who underwent the procedure of implantation with transvenous and thoracotomic approaches. 86 patients were studied: 70 patients had the LV electrode implanted transvenously, and 16 patients had mini-thoracotomy. During the follow-up period (on average of 16.4 months), significant benefits were registered in the surgical group of patients, in particular, the stimulation threshold was more stable. The incidence of complications (35\%) associated with the LV electrode in the transvenous group was unusually high. These included the inability to place the LV electrode in an optimal position, coronary vein dissection, high thresholds or loss of stimulation capture, diaphragmatic nerve stimulation, and electrode dislocation. In the surgical group, there was one complication - a displacement of the electrode. The difference was significant in favor of surgical implantation techniques [5]

\section{Conclusion}

Thus, the authors concluded that the surgical technique of implantation of a pacemaker with MEMS-converter is safe and reliable and should be considered as an equal alternative to the transvenous method in the presence of indications.

The transvenous method of pacemaker implantation has less invasiveness, shorter hospital stay and good results, but for many patients with diagnosed bradiarrhythmias who are preparing for open-heart surgery, the surgical method of pacemaker implantation with a MEMS-converter is the method of choice.

\section{Funding}

The work was supported by the grant of the Ministry of Education and Science of Russian Federation on the theme: "Production of the line of miniature wireless epicardial pacemakers with MEMS-converters for the treatment of bradiarrhythmias and heart failure" №14.607.21.0192. Unique identifier of the project RFMEFI60717X0192.

\section{Conflict of interest}

The authors declare that they have no conflict of interest.

\section{References}

1. Karaskov AM, Pokushalov YeA, Turov AN. Catheter ablation: Medical technology. Novosibirsk: NNIIPK Rosmedtekhnologii, 2008; 31 p. https://www.elibrary.ru/item.asp?id=19549661.
2. Gosline AH, Vasilyev NV, Veeramani A, Wu M, Schmitz G, Chen R, et al. Metal MEMS Tools for Beating-heart Tissue Removal. IEEE Int Conf Robot Autom 2012; 10.1109/ICRA.2012.6225210. https://doi.org/10.1109/icra.2012.6225210.

3. Daubert JC, Ritter P, Le Breton H, Gras D, Leclercq C, Lazarus A, et al. Permanent left ventricular pacing with transvenous leads inserted into the coronary veins. Pacing Clin Electrophysiol 1998; 21(1 Pt 2), 239 245. https://doi.org/10.1111/j.1540-8159.1998.tb01096.x.

4. Horenstein MS, Hakimi M, Walters H 3rd, Karpawich PP. Chronic performance of steroid-eluting epicardial leads in a growing pediatric population: a 10-year comparison. Pacing Clin Electrophysiol 2003, 26(7 Pt 1), 1467-1471. https://doi.org/10.1046/i.1460-9592.2003.t011-00212.x.

5. Mair H, Sachweh J, Meuris B, Nollert G, Schmoeckel M, Schuetz A, et al. Surgical epicardial left ventricular lead versus coronary sinus lead placement in biventricular pacing. Eur J Cardiothorac Surg 2005; 27(2), 235-242. https://doi.org/10.1016/i.ejcts.2004.09.029.

\section{Authors:}

Olga L. Bockeria - MD, DSc, Professor, Corresponding Member of the Russian Academy of Sciences, Principal Researcher, Department of Surgical Treatment for Interactive Pathology, Bakoulev Scientific Center for Cardiovascular Surgery, Moscow, Russia. https://orcid.org/0000-0002$\underline{7711-8520}$

Mikhail B. Biniashvili - MD, PhD, Leading Researcher, Department of Surgical Treatment for Interactive Pathology, Bakulev National Medical Research Center for Cardiovascular Surgery, Moscow, Russia. https://orcid.org/0000-0003-2988-309X.

Tatyana G. Le - MD, Researcher, Department of Surgical Treatment for Interactive Pathology, Bakulev National Medical Research Center for Cardiovascular Surgery, Moscow. https://orcid.org/0000-0001-9523-0172.

Anna S. Satyukova - MD, Head of the Laboratory for Control of Treatment Quality, Bakulev National Medical Research Center for Cardiovascular Surgery, Moscow, Russia, https://orcid.org/0000-0001-5948-1193.

Daniyar K. Zhiengaliev - MD, Resident of the Department of Emergent Surgery of Acquired Heart Diseases, Bakulev National Medical Research Center for Cardiovascular Surgery, Moscow, Russia. https://orcid.org/00000002-9249-5674

Vladimir A. Shvartz - MD, DSc, Researcher, Department of Surgical Treatment for Interactive Pathology, Bakulev National Medical Research Center for Cardiovascular Surgery, Moscow, Russia. https://orcid.org/00000002-8931-0376. 